\title{
Management of non-cardiac chest pain: from research to clinical practice
}

\author{
R A Mayou, C M Bass, B M Bryant
}

\begin{abstract}
Background-Non-cardiac chest pain assessed by cardiologists in their outpatient clinics or by coronary angiography usually has a poor symptomatic functional and psychological outcome. Randomised trials have shown the effectiveness of specialist psychological treatment with those who have persistent symptoms, but such treatment is not always acceptable to patients and may not be feasible in routine clinical settings.
\end{abstract}

Objectives-To describe a sample of patients referred to cardiac outpatient clinics from primary care in a single health district who were consecutively reassured by cardiologists that there was not a cardiac cause for their presenting symptom of chest pain.

Design-Systematic recording of referral and medical information of patients consecutively reassured by cardiologists. $\mathrm{Re}-$ assessment in research clinic six weeks later (with a view to inclusion in a randomised trial of psychological treatment, which has been separately reported) and followed up at six months.

Setting-A cardiac clinic in a teaching hospital providing a district service to patients referred from primary care.

Patients-133 patients from the Oxfordshire district presenting with chest pain and consecutively reassured that there was no cardiac cause during the recruitment period; 69 had normal coronary angiograms and 64 were reassured without angiography.

Intervention-A subgroup ( $\mathrm{n}=56$ ) with persistent disabling chest pain at six weeks were invited to take part in a randomised controlled trial of cognitive behavioural treatment.

Main outcome measures-Standardised interview and self report measures of chest pain, other physical symptoms, mood and anxiety, everyday activities, and beliefs about the cause of symptoms at six week assessment; repeat of self report measures at six months.

Results-Patients had a good outcome at six weeks, but most had persistent, clinically significant symptoms and distress. Some found the six week assessment and discussion useful. The psychological treatment was helpful to most of those recruited to the treatment trial, but a minority $(15 \%)$ of those treated appeared to need more intensive and individual collaborative management. Patients reas- sured following angiography were compared with those reassured without invasive investigation. They had longer histories of chest pain, more often reported breathlessness on exertion, and were more likely to have previously been diagnosed as having angina, treated with antianginal medication, and admitted to hospital as emergencies.

Conclusion-These findings suggest a need for "stepped" aftercare, with management tailored according to clinical need. This may range from simple reassurance and explanation in the cardiac clinic to more intensive individual psychological treatment of associated underlying and often enduring psychological problems. Simple ways in which the cardiologist might improve care to patients with non-cardiac chest pain are suggested, and the need for access to specialist psychological treatment discussed. (Heart 1999;81:387-392)

Keywords: non-cardiac chest pain; psychological problems; cognitive behavioural treatment

Chest pain is the most common reason for referral to district cardiac services. Over half of the patients referred are diagnosed as having chest pain not caused by ischaemic heart disease or any other major physical cause. ${ }^{1}$ In a district service, the majority of patients do not undergo invasive investigation and a diagnosis of the pain as non-cardiac is usually made by the cardiologist following history, physical examination, and other investigations.

Patients reassured that they do not have heart disease are known to have a poor overall outcome in terms of symptoms, distress, disability, and continuing concern about heart disease. ${ }^{2}$ Few are given explanations of the minor medical problems which may underlie symptoms. ${ }^{3}$ Some patients recover following straightforward reassurance but many have a poor outcome and appear to need extra help. These patients with persistent symptoms are usually regarded as difficult to treat. ${ }^{1}$ We have previously reported the characteristics and three year outcome of consecutive referrals to a cardiac clinic.

It is well known that it is difficult to apply conclusions from randomised controlled trials carried out by expert therapists with carefully selected subjects, to the routine delivery of care to large numbers of patients in everyday circumstances. We have shown that a specialist intervention by a psychologist (sessions of cognitive behavioural treatment) was effective 
for patients with chronic symptoms who were recruited in primary care. ${ }^{4}$ We have recently replicated this finding for patients with persistent symptoms following attendance at a cardiac clinic. ${ }^{5}$ In the second study less than one third $(28 \%)$ of the patients we assessed were both suitable for and willing to take part in this trial. ${ }^{5}$

The randomised trial raises wider questions about effective and acceptable care following negative investigation and the acceptability of psychological explanations of symptoms. How can appropriate extra care be provided to those who need it? Our research suggests that it would be useful to think of a process of "stepped" care in which all patients are offered appropriate, simple care providing clear explanation of symptoms and advice, with extra help being offered to the minority who have severe problems or fail to improve during follow up. We need to define detailed procedures for improved routine care. In this paper we describe findings from the total consecutive sample of patients considered for our cardiac clinic treatment trial, subsequently reassured by the cardiologist that their chest pain was non-cardiac. Regional tertiary referrals from physicians and others outside the health district were not included.

We first considered a subsidiary question: do patients investigated by angiography have a different outcome and continuing treatment needs to those who do not undergo invasive investigation?

Next, in the principal section of the results, we characterised the various subgroups of the whole sample of patients according to their treatment needs: those satisfied by negative investigation and simple reassurance; those with more persistent symptoms and distress at follow up; and those with multiple psychiatric and physical problems.

Inevitably, problems of missing data limit presentation and interpretation. The practical issues are of great importance, however, if research findings are going to lead to improvements in care for patients with non-cardiac chest pain and other functional symptoms. Our data from a representative sample who were offered further assessment in order to recruit for a randomised trial provides data for systematic description of characteristics and treatment needs.

\section{Methods}

We studied consecutive patients over a one year period who were referred to a specialist cardiac clinic for diagnosis of the principal complaint of chest pain and who were aged 18-65 years, lived in Oxfordshire, and whose investigations were negative for ischaemic heart disease. Each patient was sent a letter from the cardiologist, repeating the reassurance that there was no cardiac cause for their symptoms and offering them a follow up appointment at a research clinic for non-cardiac chest pain six weeks after discharge from hospital/outpatient clinic. There were two groups of patients in terms of extent of investigation: 69 consecutive patients whose investigations included angiography and who were found to have normal coronary arteries and then reassured (angiogram group), of whom 50 attended at six weeks; 64 consecutive new patients attending a cardiology outpatient clinic who were reassured they did not have ischaemic heart disease without undergoing angiography (outpatient group), of whom 40 attended at six weeks.

The research clinic was situated in the main outpatient area of the same general hospital. Patients were recruited over a period of 16 months (from August 1991 to November 1992). The assessment consisted of semistructured interviews lasting about 20 minutes each, with a consultant psychiatrist and a research interviewer, to obtain baseline demographic and medical information, and information about social impairment in the domains of leisure activities, work, and family. Measures of mood, mental state, and beliefs about causes of symptoms were also obtained from interview questions, and from the structured clinical interview for the Diagnostic and Statistical Manual of Mental Disorders, third edition, revised (DSM-IIIR), ${ }^{6}$ and self report questionnaires including the brief symptom inventory, ${ }^{7}$ the Whitely index ${ }^{8}$ and a hyperventilation check list. ${ }^{9}$

Follow up assessments repeating these measures were carried out three and six months later for the 37 patients recruited into the treatment trial. The remainder, including those who initially refused the assessment, were sent a postal follow up questionnaire at the six month point, asking about current symptoms and treatment, satisfaction with medical services, and causes of the symptoms. They again completed the brief symptom inventory.

Follow up information was obtained from interview for all except three of the 37 who took part in the treatment trial (20 angiogram group, 17 outpatient group). The results of this study have been published elsewhere. ${ }^{5}$ A postal questionnaire was sent to 88 of the 96 remaining subjects (eight did not wish to be contacted again), and 48 (55\%) of them completed this.

\section{Results}

Figure 1 summarises the principal categories of patients described in this paper. The presentation of results begins with a comparison of the patients with and without normal coronary arteries. The principal section of the Results is a description of each of the categories in fig 1 .

DO THOSE INVESTIGATED BY CORONARY ANGIOGRAPHY HAVE A DIFFERENT OUTCOME AND TREATMENT NEEDS FROM THOSE WHO ARE REASSURED WITHOUT INVASIVE INVESTIGATION? The sample contained two main groups of subjects who completed assessment of chest pain and were reassured they did not have heart disease during the study period; those who had outpatient investigations only $(n=64)$, and those who had been admitted for coronary angiography $(n=69)$.

The two groups were demographically similar, apart from the outpatient group being much younger and less likely to be married, but were medically very different (table 1 ). The angiogram group of patients had longer 


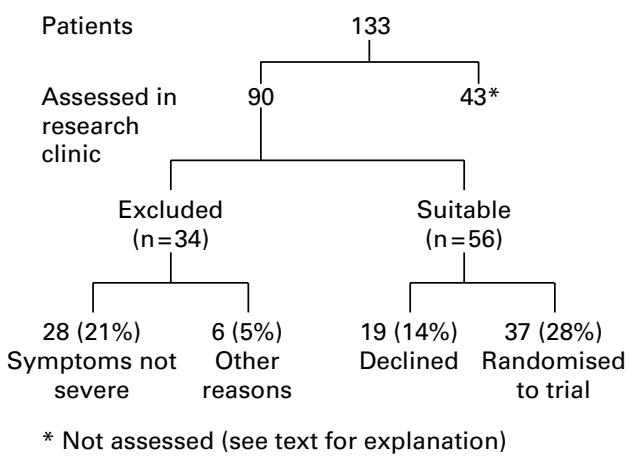

Figure 1 Flow chart of consecutive patients attending research clinic during 16 month period.

histories of chest pain, more often reported breathlessness and pain on exertion, were more likely to have been diagnosed as having angina, treated with antianginal medication and admitted to hospital as emergencies, and to have had abnormal hospital test results. The groups did not differ with regard to having a family history of heart disease, palpitations, other physical illnesses, previous psychiatric problems, or smoking.

At the six week research assessment, those investigated by angiography reported more frequent symptoms and rated themselves as more limited: 27 of 50 (54\%) said they were moderately or very limited by their symptoms, compared with 9 of $40(23 \%)$ of the outpatients $(p<0.01)$. They were also more likely to report longstanding social problems. The groups did not differ in the severity of their chest pain symptoms, their subjective feelings of distress, or in attribution of their symptoms to heart disease or stress. On the rating scales the only differences between the two groups were significantly higher mean (SD) scores in the normal coronary angiogram group on the brief symptom inventory somatisation subscale (0.93 (0.69) v $0.56(0.49), \mathrm{p}<0.01)$ and the hyperventilation checklist $(14.30 \quad(9.20) v$ 10.47 (7.9), $\mathrm{p}<0.05)$.

Table 1 Characteristics of subjects taken from hospital notes

\begin{tabular}{|c|c|c|c|}
\hline & $\begin{array}{l}\text { Normal coronary } \\
\text { angiogram }^{*}(n=69)\end{array}$ & Outpatients $(n=64)$ & Significance \\
\hline Mean (SD) age (years) & $51.65(8.73)$ & $43.95(10.78)$ & $\mathrm{p}<0.001$ \\
\hline \multicolumn{4}{|l|}{ Sex } \\
\hline Male & $38(55 \%)$ & $40(63 \%)$ & \\
\hline Female & $31(45 \%)$ & $24(38 \%)$ & \\
\hline \multicolumn{4}{|l|}{ Marital state } \\
\hline Married & $58(84 \%)$ & $39(61 \%)$ & \\
\hline Not married & $6(9 \%)$ & $14(22 \%)$ & $\mathrm{p}<0.02$ \\
\hline Not known & $5(7 \%)$ & $11(17 \%)$ & \\
\hline \multicolumn{4}{|l|}{ History of chest pain } \\
\hline Never & $2(3 \%)$ & & \\
\hline$<6$ months & $28(41 \%)$ & $31(48 \%)$ & \\
\hline $6-23$ months & $19(28 \%)$ & $27(42 \%)$ & $\mathrm{p}<0.02$ \\
\hline$>2$ years & $19(28 \%)$ & $6(9 \%)$ & \\
\hline \multicolumn{4}{|c|}{ Emergency admission for chest pain } \\
\hline No & $40(59 \%)$ & $60(94 \%)$ & \\
\hline Yes & $28(41 \%)$ & $4(4 \%)$ & $\mathrm{p}<0.001$ \\
\hline \multicolumn{4}{|l|}{ Antianginal medication in past } \\
\hline No & $34(48 \%)$ & $49(77 \%)$ & \\
\hline Yes & $33(50 \%)$ & $12(19 \%)$ & $\mathrm{p}<0.001$ \\
\hline Yes (blood pressure only) & $1(2 \%)$ & $3(5 \%)$ & \\
\hline \multicolumn{4}{|c|}{ General practitioner mentioned angina as cause } \\
\hline No & $33(48 \%)$ & $46(72 \%)$ & \\
\hline Yes & $36(52 \%)$ & $18(28 \%)$ & $\mathrm{p}<0.01$ \\
\hline \multicolumn{4}{|l|}{ Previous episodes } \\
\hline No & $56(82 \%)$ & $52(81 \%)$ & \\
\hline Yes & $12(18 \%)$ & $12(19 \%)$ & NS \\
\hline
\end{tabular}

${ }^{\star}$ Hospital notes missing for one person.
Surprisingly, the longer term outcomes of the two groups were more similar. Subjective severity of symptoms at six months was significantly worse in the angiogram group, but otherwise the groups did not differ. Outcome, whether or not subsequently treated or untreated, was better than has been previously consistently reported for those with normal coronary angiograms. ${ }^{10}$

CAN WE IDENTIFY SUBGROUPS IN TERMS OF FOLLOW-UP OUTCOME AND TREATMENT NEEDS? Having compared the two groups defined in terms of whether or not they were investigated by angiography, we now describe subjects in terms of subgroups categorised according to their chest pain status at our six week research clinic review (summarised in fig 1).

Patients who declined or did not attend the six week assessment $(n=43)$

Two thirds of the cohort (90 patients) attended the six week research clinic for assessment$56 \%$ of the angiogram group $(n=50)$ and $44 \%$ of the outpatients $(n=40)$. Most of those spoken to on the telephone said they did not see a need for further attendance as they now had no symptoms or felt a great deal better; a few appeared to be more disabled but declined the offer of research clinic follow up. The reasons for non-attendance by 43 subjects were as follows: 20 subjects had either no pain or infrequent pain which they were coping with satisfactorily; six patients refused assessment because they were too busy or it was too far to travel; two specifically refused because the research clinic sounded "too psychological"; seven were excluded as unsuitable for our trial because of other physical or psychological problems for which most were already receiving medical care; 10 patients were sent appointments but did not attend and could not be contacted by post or telephone.

Patients who declined the follow up assessment or did not attend for appointments had shorter histories of symptoms, reported less varied and less severe symptoms to the cardiologist, and were less likely to be on current antianginal medication. The six month follow up indicates a good six month outcome for the 14 subjects who replied (table 2).

There were, however, a small number of subjects in this group who were suspicious of the research but who appeared to be at risk of a poor outcome and who might have benefited from extra help, in addition to the seven patients who had other physical or psychological problems involving continuing medical care elsewhere.

\section{Patients with infrequent symptoms at follow up} $(n=34)$

Twenty eight $(22 \%)$ of those who were assessed at six weeks described either no $(n=17)$ or infrequent $(n=12)$ symptoms (pain less than once a week) or disability which did not satisfy the criteria for recruitment to our treatment trial. While usually not greatly concerned by their symptoms, most were grateful for the extra research assessment which provided an 
Table 2 Outcome at six months classified according to participation in the assessment and treatment trial

\begin{tabular}{|c|c|c|c|c|c|c|}
\hline & \multirow[t]{2}{*}{ Not assessed } & \multicolumn{4}{|l|}{ Assessed } & \multirow[b]{3}{*}{ Significance $t^{2}$} \\
\hline & & \multirow[b]{2}{*}{$\begin{array}{l}\text { No symptoms or symptoms } \\
<1 \times \text { week } n(\%)\end{array}$} & \multicolumn{3}{|c|}{ Symptoms at least $1 \times$ week } & \\
\hline & $\begin{array}{l}\text { Non-attenders eligible } \\
\text { for inclusion } n(\%)\end{array}$ & & $\begin{array}{l}\text { Refused trial } \\
n(\%)\end{array}$ & $\begin{array}{l}\text { Treatment group } \\
n(\%)\end{array}$ & $\begin{array}{l}\text { Control group } \\
n(\%)\end{array}$ & \\
\hline Number of patients & 14 & 20 & 10 & 20 & 14 & \\
\hline \multicolumn{7}{|l|}{ Severity of symptoms } \\
\hline Moderate/severe/very severe & $4(29)$ & $4(20)$ & $4(40)$ & $10(50)$ & $9(64)$ & $\mathrm{p}<0.1$ \\
\hline \multicolumn{7}{|l|}{ Distress of symptoms } \\
\hline Moderate/great & $3(21)$ & $1(5)$ & $1(10)$ & $10(50)$ & $6(43)$ & $\mathrm{p}<0.01$ \\
\hline Mean BSI global score & 0.32 & 0.35 & 0.58 & 0.38 & 0.26 & NS \\
\hline
\end{tabular}

BSI, brief symptom inventory.

opportunity for discussion of the causes and prognosis of non-cardiac chest pain. A further six patients were excluded at this stage because of cardiac or psychiatric illnesses.

Most patients with few symptoms at six week assessment felt reassured and were optimistic. They had lower scores on psychometric scales. Most reported good outcomes on all measures at six months.

Patients with frequent symptoms at follow up $(n=56)$

Just under half of the patients were seen at six months and were assessed as having continuing symptoms and disability sufficient to satisfy inclusion criteria for our treatment trial.

Patients who refused extra treatment $(n=19)$

Patients who satisfied the trial criteria in terms of frequency of chest pain and limitation of everyday activities were all offered the opportunity of entering the treatment trial and the trial rationale was explained to them. The 19 patients who declined were rated as having somewhat less severe symptoms than those who were eventually recruited and scored lower on the psychometric scales. There appeared to be two main reasons for declining. Eight patients were grateful for the explanation and said they now understood their symptoms. They could manage to cope with their problems without further assistance. The subjects generally reported a good subsequent outcome. Eleven patients found the rationale and the practical demands of a psychological intervention unacceptable. Several said that they felt our treatment would not be helpful with symptoms that could only have a physical cause and required physical treatment. Most had a poor subsequent outcome.

Patients who agreed to take part in the treatment trial $(n=37)$

The outcome of the treatment trial for 20 treated subjects and 17 controls has been reported elsewhere. ${ }^{5}$ There was clear evidence of substantial improvement in 14 of the 16 patients who attended treatment, and in a number of further patients who had some treatment but failed to complete the full sessions. Four who dropped out before receiving any treatment were unimproved at six month follow up. The control group had a poorer outcome with no improvement in mean scores for outcome variables.

While treatment was usually effective for those who were interested and attended, it was evident that a minority had complex psychological and physical treatment needs.

\section{Discussion}

It is well known that subjects recruited from routine clinical services for randomised controlled clinical trials are usually atypical. Demonstration of a successful treatment for persistent non-cardiac chest pain raises questions about delivery in routine care which are difficult to answer. This paper examines these issues and their clinical significance for consecutive patients assessed by cardiologists as having non-cardiac chest pain.

STUDY LIMITATIONS

The findings from this particular sample (alongside evidence from our previous research) enable recommendations for changes in routine care. Before discussing these it is important to note that our consideration of outcome is complicated by a number of overlapping factors. Firstly, baseline and six month follow up data were incomplete despite strenuous efforts by post and telephone. A substantial minority of patients felt that they did not need to attend a further follow up and response rates to postal follow up were low. Even so, medical information was available on all subjects and there was telephone contact with many of those who felt that participation was unnecessary. Secondly, a minority of patients received a treatment designed to improve outcome. Thirdly, the assessment interview and explanation of the treatment rationale may have had a therapeutic effect for the two thirds who attended the six week follow up interview at the research clinic. Similar findings have been reported by others. ${ }^{11}$

Despite these problems, always encountered in this type of clinical research, we do have information on all the consecutive patients. This provides a solid basis for categorising patients in terms of their clinical characteristics and for drawing conclusions about their rather different requirements for subsequent care.

\section{ROLE OF ROUTINE FOLLOW UP}

At least one third of patients, especially those with milder and shorter duration of symptoms, improve following negative investigation and simple reassurance. They often feel further hospital attendance is unnecessary and appear to have a generally favourable longer term outcome. The six month follow up shows that a small number of people who were satisfied and 
confident initially reported later symptoms and limitations. These patients might have benefited from a review appointment by the same cardiologist who carried out the initial assessment two to three months after their clinic appointment.

EXTRA NEEDS OF THOSE UNDERGOING INVESTIGATIONS, WHICH INCLUDED ANGIOGRAPHY

Patients investigated by angiography had more severe symptoms and were more likely to have been told they had heart disease and be treated as such before angiography. They require better preparation before angiography and extra information at the time of the investigation and at follow up. In those patients with atypical symptoms in whom a negative angiogram is anticipated, discussion of the likelihood and possible meaning of a negative test is likely to be beneficial before the procedure. The additional use of fact sheets and audiovisual aids may be valuable but requires further evaluation (see Lloyd and colleagues ${ }^{12}$ for the difficulties with this approach). These "procedural" aspects of angiography offer research possibilities for cardiologists.

Many major cardiac centres receive substantial numbers of tertiary referrals from other physicians in their region. Provision of information and good communication will be especially important for patients who may have travelled a considerable distance and who may or may not be followed up by the referring doctor.

\section{NEED TO PROVIDE AN EXPLANATION}

Patients frequently comment on a need for explanation for their symptoms in addition to reassurance that they do not have heart disease. There are numerous non-cardiac disorders that need to be considered in the management of these patients. Oesophageal disorders are relatively common in these patients, but their relevance to the experience of chest pain is uncertain. ${ }^{13}$ However, acid reflux is common and some patients respond well to $\mathrm{H}_{2}$ receptor antagonists or proton pump inhibitors. It has been shown that as many as one third are helped by a trial of proton pump inhibitors. ${ }^{14}$ If there is no evidence of conspicuous psychological abnormality, oesophageal function tests may be indicated. These may reveal a small number of patients with motility disorders or acid reflux unresponsive to first line medication. These may need specialist gastroenterological referral.

NEEDS OF THOSE WITH MILD SYMPTOMS AT FOLLOW UP

Patients whose symptoms are rated as mild at follow up are usually optimistic, grateful for the further discussion of their symptoms, and have a good outcome. A six week appointment provides an opportunity for reinforcing explanations and discussing issues which are difficult to address during the interview with the cardiologist. Such patients often feel that attendance for further treatment is not necessary but a fur- ther review within three to six months would be appropriate to confirm the improvement.

NEEDS OF THOSE WITH PERSISTENT SYMPTOMS AT FOLLOW UP

For those with persistent chest pain, limitation of activities, and evidence of psychological distress or abnormal illness beliefs-for example, conviction that angina is present despite evidence to the contrary-psychological treatment may be indicated. Cognitive behavioural treatment of the type that we have shown to be useful is usually effective. ${ }^{4}$ Other patients may require individualised psychological, social or psychiatric treatment. It would be appropriate to offer such a patient a routine follow up appointment in the clinic itself or, if properly coordinated, within primary care. There is probably an advantage in suggesting the former so that any further treatment can then be seen to be clearly initiated by the cardiologist as part of standard treatment of a common medical problem. Psychological and other interventions would be more acceptable in this context than as a special further referral to psychiatric and psychological services from primary care.

ACCEPTABILITY OF PSYCHOLOGICAL TREATMENT A small proportion of patients with severe and limiting symptoms find a psychological approach unacceptable or inconvenient and refuse or drop out of treatment. It is probable that a more collaborative follow up within cardiology would be successful in involving these patients in treatment.

CLINICAL IMPLICATIONS

It is not surprising that we found considerable variation in the clinical needs of a sample defined in terms of a presenting physical symptom. Subjects vary not only in terms of the frequency and severity of symptoms and associated disability, but also in their concerns and beliefs, and in their needs for explanation and treatment of their physical and psychological problems. The heterogeneity of consecutive attenders, their characteristics at referral, and their varied outcomes supports the need for the flexible and "stepped" approach to management outlined above.

The cardiologist working in a busy outpatient clinic may require access to additional resources if he or she is to provide adequate management of patients with non-cardiac chest pain. One possibility is to employ a specialist cardiac nurse who has received additional training in the management of these clinical problems; this individual could undertake explanation and simple psychological intervention and routine follow up in a separate part of the cardiac outpatient clinic. A review appointment for all patients with a cardiac nurse either in the cardiac clinic or with the doctor in primary care would be valuable in identifying patients with recurrent or persistent symptoms. Identifying the treatment requirements at an earlier stage in the evolution of the disorder is essential.

Our findings, using a wide range of standardised measures, should be considered alongside 
increasing evidence of the value of specialist rapid access chest pain clinics. ${ }^{15} 16$

Some patients will require more specialist treatment. For this group it would be important for the cardiac department (possibly the cardiac nurse) to collaborate with psychology/ psychiatry services experienced in dealing with these clinical problems.

The increasing interest in rapid access, one stop cardiac clinics for the assessment of chest pain may lead to earlier recognition of patients requiring treatment for coronary artery disease. At the same time, it is important to be aware that the prevalence of continuing chest pain two years after referral is higher in those with non-cardiac pain than in those with established ischaemic disease. ${ }^{15}$ The improvements in advice and management that we have outlined may have major benefits for these patients, including a reduction in their subsequent use of medical resources.

This paper is concerned with district referrals from primary care. The general principles that we have discussed will require different emphases for centres with large numbers of tertiary referrals and in other districts which do not provide specialised angiography and other investigation, and which refer to regional centres.

1 Mayou R, Bryant B, Forfar C, et al. Non-cardiac chest pain and benign palpitations in the cardiac clinic. Br Heart $f$ 1994;72:548-53.
2 Bass C, Mayou RA. Chest pain and palpitations. In: Mayou RA, Bass C, Sharpe M, eds. Treatment of functional somatic symptoms. Oxford: Oxford University Press, 1995:328-52. 3 Chambers J. Chest pain: heart, body or mind? $\mathcal{F}$ Psychosom Res 1997;43:161-5.

4 Klimes I, Mayou RA, Pearce MJ, et al. Psychological treatment for atypical non-cardiac chest pain: a controlled evaluation. Psychol Med 1990;20:605-11.

5 Mayou R, Bryant B, Sanders D, et al. A controlled trial of cognitive behavioural therapy for non-cardiac chest pain. Psychol Med 1997;27:1021-32.

6 Spitzer RL, Williams JBW, Gibbon M, et al. User's guide to the structured clinical interview for DSM-IIIR. Washington: American Psychiatric Press, 1990 .

7 Derogatis LR, Melisaratos N. The brief symptom inventory: an introductory report. Psychol Med 1983;13:595-605.

8 Pilowsky I. Dimensions of hypochondriasis. Br F Psychiatry 1967;113:89-93.

9 Pickles C. Cognitive factors in hyperventilation [Msc thesis]. London: University of London Institute of Psychiatry, 1987.

10 Potts SG, Bass CM. Psychological morbidity in patients with chest pain and normal or near-normal coronary arteries: a long-term follow-up study. Psychol Med 1995;25: arteries:

11 Speckens AEM, Van Hemert AM, Spinhoven P, et al. Cognitive behavioural therapy for medically unexplained physical symptoms: a randomised controlled trial. BMF 1995;311:1328-32.

12 Lloyd G, Cooper A, Jackson G. Information delivery: the provision of written information for patients following coronary angiography and post-discharge management. International fournal of Clinical Practice 1997;51:387-8.

13 de Caestecker J. Oesophageal chest pain: an update. In: Kaski J, ed. Chest pain with normal coronary angiograms: pathogenesis, diagnosis and management. Philadelphia: Kluwer Academic, 1998.

14 Chambers J, Cooke R, Angiannsah A, et al. Beneficial effects of omeprazole in patients with chest pain and normal corof omeprazole in patients with chest pain and normal cor-
onary anatomy: preliminary findings. International Cardiolonary anatomy.
ogy. [In press.]

15 Jain D, Fluck D, Sayer JW, et al. One-stop chest pain clinic can identify high cardiac risk. $\mathcal{F} R$ Coll Physicians Lond 1997;31:401-4.

16 Newby DE, Fox KAA, Flint LL, et al. A 'same day' direct-access chest pain clinic: improved management and reduced hospitalization. QfM 1998;91:333-7. 